\title{
MATHEMATICAL ANALYSIS OF RECEIVER FOR A PARABOLIC DISH COLLECTOR
}

\author{
S. Abhiram \\ ME (Thermal Engineering) Student, \\ Chaitanya Bharathi Institute of Technology, Hyderabad, India \\ Dr. M.V.S Murali Krishna \\ Professor, Chaitanya Bharathi Institute of Technology, Hyderabad, India
}

\begin{abstract}
Solar energy is an energy derived from sun. The intensity of the Sun's rays gets reduced as it travels through the atmosphere. New and enhanced version of receiver in Parabolic Dish collector helps in increasing the output, which is created by sandwiching of two different materials rather than that of one. Study is conducted by comparing steady heat transfer rate in terms of their thermal conductivity. Comparatively, this enhanced version of receiver in Parabolic Dish Collector is replaced by two materials and sandwiching them together. With Transient Thermal analysis, rate of temperature along the inner walls of receiver are predicted and accordingly the need for coupling of materials is studied.
\end{abstract}

Keywords: Parabolic dish collector, receiver, transient.

Cite this Article: S. Abhiram and M.V.S Murali Krishna, Mathematical Analysis of Receiver for a Parabolic Dish Collector, International Journal of Mechanical Engineering and Technology (IJMET), 12(7), 2021, pp. 17-22.

https://iaeme.com/Home/issue/IJMET?Volume=12\&Issue=7

\section{INTRODUCTION}

Solar energy is one of renewable energy sources, where the source of power production never exhausts. Sun being source, sun rays travel to Earth's, where energy is generated. Energy generating methods by [1]Mamodiya et al., suggested various methods of power production. Every method has its own merits and demerits. With carefully consideration of resource availability and energy requirements one among these methods are considered. The present study deals with the generation of heat energy by Parabolic dish concentration method. As name suggest, a parabola dish is considered, and a receiver is placed at its focal point. Here the intensity of solar beams is focused for utilization either in the form heat or electricity output.

To achieve higher efficiency, the geometry of collector, position of receiver which were analysed by [2]Ali et al., along with efficiency on power production by varying geometry. Like the geometry of collector, geometry of receiver also plays an important role. The directions of fluid inlet and outlet studied by [3]Cherif et al., depending on the height of the receiver to that 
of base, direction of fluid flow is to be selected for optimal results. For better understand of focal length, positioning of receiver [4]Saleh et al., used ray tracing methods by which exact focal length is obtained, black coated steel receiver is used for energy production. Black coated material is for high absorptivity for better results. But [5]Zwinkels, described about the radiation losses occurs due to rise in black body temperature.

Estimation of power conversion from collector to receiver studied by [6]Alarcon et al. where collector geometry is formulated, along with optical losses, convectional and radiational losses are considered accordingly a prototype was created and analysed. Focal position is analysed by [7]Pavlovic et al., using TracePro software and a spiral absorber inside receiver for better thermal energy generation. Using another software Energy3D [8]Mandhare et al., energy for cities was calculated, from which the collector as well as receiver geometry is generated.

Properties of materials are considered from $[10,11]$ for this study.

\section{RECEIVER DESIGN}

Four types of arrangements are considered for this study.

\subsection{High Thermal Conductivity Receiver, Type 1}

A single material having high thermal conductivity is taken for power generation. For this study, the time required for a steady state is noted and compared. The considered material is aluminium.

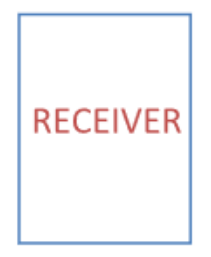

Figure 1 Representation of Uniform Material

\subsection{Low Thermal Conducting Receiver, Type 2}

A single material having low thermal conductivity is selected for power generation. For this study, the time required for a steady state is noted and compared. Same as that of fig. 1. The considered material is lead.

\subsection{Sandwich Type, Type 3}

In this type, two low thermal conductivity material and three high thermal conductive materials are used as shown in fig.2, here lead dominate the solar concentration while aluminium dominates thermal energy production. By having the larger contact with the fluid medium, high energy is transferred causing less time to stabilize and as low thermal conductive material face the solar concentration, even a sudden drop in intensity will not immediately affect the fluid temperature. Rays representing concentrated rays falling on receiver from collector. In fig.2, rays representing concentrated rays falling on receiver from collector. 


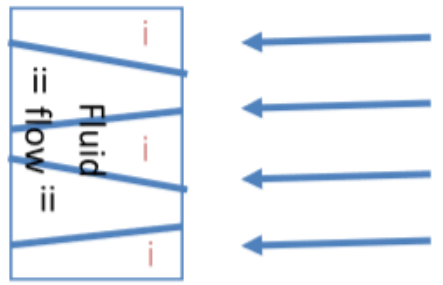

Figure 2 Representation of Type 3

$i$ - low thermal conductive material

ii-high thermal conductive material

\subsection{Sandwich Type, Type 4}

In this type, two high thermal conductivity material and three low thermal conductive materials as shown in fig.3, here aluminium dominate the solar concentration while lead dominates thermal energy production. By having the larger contact with the fluid medium, low energy is transferred causing more time to stabilize and as high thermal conductive material face the solar concentration, even a slight drop in intensity will instantly affect the fluid temperature. In fig.3, rays representing concentrated rays falling on receiver from collector.

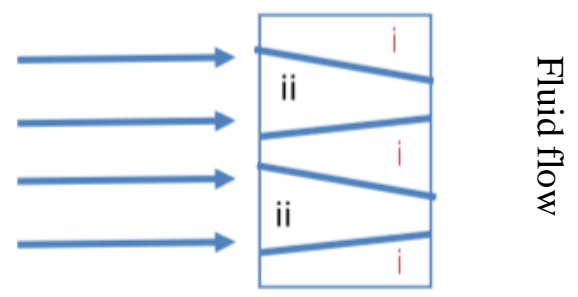

Figure 3 Representation of Type 4

$i$ - low thermal conductive material

ii-high thermal conductive material

\section{DESIGN PROCEDURE}

\subsection{Initial Calculation}

The solar intensity is calculated, depending on the geometrical values of collector and optimal receivers' geometry and its position is calculated and temperature of receiver is obtained.

Table 1 shows the geometrical values for aperture diameter, focus of parabolic curve and radius of cylinder.

Table 1 Initial Values

\begin{tabular}{|l|c|}
\hline \multicolumn{1}{|c|}{ Nomenclature } & Value(S.I Units) \\
\hline Aperture Diameter, Da & $\mathbf{1 . 5 0 1}$ \\
\hline Focal Length, $\mathbf{F}$ & $\mathbf{1 . 4 1 9}$ \\
\hline Radius of aperture cylinder, $\mathbf{r}$ & $\mathbf{0 . 0 1 5}$ \\
\hline Thickness of receiver, $\mathbf{x}$ & $\mathbf{0 . 0 0 5}$ \\
\hline Velocity of fluid(water), $\mathbf{V}$ & $\mathbf{1}$ \\
\hline Density of fluid, $\boldsymbol{\rho}$ & $\mathbf{1 0 0 0}$ \\
\hline Specific heat of fluid, $\mathbf{C p}$ & $\mathbf{4 1 9 0}$ \\
\hline
\end{tabular}




\subsubsection{Assumptions}

As the sunlight reaches earth surface, an estimation of $\mathbf{2 0 \%}$ loss is expected and solar constant value is taken as $1360 \mathrm{~W} / \mathrm{m}^{2}$.

Height of the receiver is taken such a way that it is completely covered in concentrated rays.

Table 2 shows the values obtained from tab.1 by using formulae (1-6) in appendices are:

Table 2 Obtained Values

\begin{tabular}{|l|c|}
\hline \multicolumn{1}{|c|}{ Nomenclature } & Value \\
\hline Area of aperture, $\mathbf{A a}\left(\boldsymbol{m}^{\mathbf{2}}\right)$ & $\mathbf{1 . 7 6 9 5}$ \\
\hline Area of receiver, $\mathbf{A r}\left(\boldsymbol{m}^{2}\right)$ & $\mathbf{0 . 0 0 0 3 8 7 9 2}$ \\
\hline Height of receiver, $\mathbf{h}(\mathbf{m})$ & $\mathbf{0 . 0 0 2 0 5 8}$ \\
\hline Concentration factor, $\mathbf{C}$ & $\mathbf{4 5 6 1 . 4 7}$ \\
\hline Heat generation on receiver, $\mathbf{h g}(\mathbf{W})$ & $\mathbf{2 4 0 6 . 5 2}$ \\
\hline
\end{tabular}

\section{METHODOLOGY}

Mathematical analysis of two different materials is analysed. From Fourier's equation, the thermal conductivity value is substituted and are waged with different materials.

$$
\dot{q}=K \times \nabla T
$$

Where,

$\mathrm{K}$ is material thermal conductivity.

$\nabla T$ is the temperature gradient per unit cross sectional area.

Different types of material configurations have different effects. The value alone shows huge difference in eq. 1.

Procedure for finding the efficient receiver is by mathematically analysing each type individually.

After testing of individual type are compared and will be decided for the perfect receiver type.

\section{TABULATED VALUES OF RECEIVER}

Two different material considered are Aluminium and Lead, with aluminium being high thermal conductivity and lead being low thermal conductivity.

The values are taken from [10,11]. Time comparison for steady state is evaluated using eq. 1 $\&$ formula (7) in appendices, and are compared in the following table 3.

Table 3 Comparison of receiver types

\begin{tabular}{|c|c|}
\hline Type & Time(s) \\
\hline Type 1 & 8.2 \\
\hline Type 2 & 55 \\
\hline Type 3 & 12.59 \\
\hline Type 4 & 18.13 \\
\hline
\end{tabular}

\section{RESULTS AND DISCUSSION}

First type, where the complete high thermal conductive material is used. Single material corresponds to easier results, upon calculated it is the fastest possible method for achieving high temperature, subsequently resulting in high thermal energy production. 
Second type, where the complete low thermal conductivity material is used. Single material corresponds to easier results, upon calculating it is discovered that slowest possible method for achieving steady temperatures which is less when compared to that of first type. Even though the duration is large implies sudden change in the solar intensity is not at all effects the fluid output. This is more economical and reliable when compared to that first type which drops even faster with fall of intensity.

Third type, where the combination of two materials is used, here analysing the receiver is difficult. Because of having two types of materials are sandwiched to get good economic and reliable results.

Similarly goes with fourth type, as the compounds both are same and time consuming is constant. But the fourth is better when compared to third as the thermal conduction interacting with fluid flow is more, resulting in high temperature but with slight sensible to temperature change when compared that of other.

\section{CONCLUSIONS}

According to the results, I conclude by saying that type 4 receiver is economical, reliable and profitable where thermal energy production is necessary, whereas for warm fluid availability where the need for high temperatures is not required but maintaining those temperature for long durations if necessary, type 3 is selected. According to the application, the best type of receiver is used for thermal energy utilization.

\section{ACKNOWLEGEMENT}

Authors are thankful to the authorities of Chaitanya Bharathi Institute of Technology, Hyderabad, for providing necessary facilities for carrying out this work..

\section{APPENDICES}

\section{Formulas}

- Area of aperture, $\mathrm{Aa}=\pi \times D a^{2} / 4$

- Area of receiver, $\mathrm{Ar}==2 \times \pi \times r \times h$

- Height of receiver, $\mathrm{h}=2 \times \operatorname{cosec}\left(\frac{\alpha}{2}\right)$

- Concentration factor, $\mathrm{C}=\frac{A a}{A r}$

- $\quad$ Intensity reaching earth, $\mathrm{I}=I_{s c} \times 0.8$

- Power generation from solar rays, $\dot{Q}=I \times C \times A r$

- Parallel connected thermal conductivity, $\mathrm{Kp}=\frac{K 1 \times A 1+K 2 \times A 2}{A}$

\section{REFERNCES}

[1] Udit Mamodiya, Dr. Neeraj Tiwari, 2019. ”Electricity Generation Through Solar Energy Concept and Its Mechanism” JTGRS, Volume 21 Issue 5, ISSN: 0374-8588

[2] Imhamed M. Saleh Ali, S.Reddy K,Tadhg S O'Donovan, Tapas K Mallick, 2012, ”Optical Performance Of Circular and Elliptical 3-D Static Solar Concentrators ", preceded at World Renewable Energy Congress, Colorado,USA, Doi: 10.13140/2.1.4550.0166 
[3] Hiba Cherif, Anissa Ghomrassi, Jalila Sghaier, Hatem Mhiri, Philippe Bournot, 2019, "A Receiver Geometrical Details Effect on a Solar Parabolic Dish Collector Performance", ELSEVIER, Energy reports, Volume 5, pp. 882-897, doi: 10.1016/j.egyr.2019.07.010 .

[4] Imhamed M. Saleh, Khalifa Khalifa, Mohamed Bughazem and Nabil Algharbi, 2016, "Developments in Parabolic Solar Dish Concentrator for Enhanced System Efficiency of Steam Generation", SUSJ, Volume 6, Issue 1.

[5] Zwinkels J.C.,2015, “Blackbody and Blackbody Radiation”. In: Luo R. (eds) Encyclopedia of Color Science and Technology. Springer, Berlin, Heidelberg. Doi: https://doi.org/10.1007/978-3-642-27851-8_370-1”.

[6] Jorge Alexander Alarcón , Jairo Eduardo Hortúa, Andrea López G, "Design and Construction of a Solar Collector Parabolic Dish for Rural Zones in Colombia" TECCIENCIA, DOI: http:/dx.doi.org/10.18180/tecciencia.2013.14.2

[7] Saša R. Pavlovic, Velimir P. Stefanovic, 2015, "Ray Tracing Study of Optical Characteristics of the Solar Image in The Receiver for a Thermal Solar Parabolic Dish Collector", Journal of Solar Energy, vol. 2015, Article ID 326536, 10 pages, Doi: https://doi.org/10.1155/2015/326536.

[8] Shravan Mandhare, Ramola Sinha, 2020, "Thermal Analysis of Parabolic Dish Receiver System and Energy Analysis by Using Energy 3D Software", IJERT, Volume 9 Issue 08, ISSN: 22780181 .

[9] Ansys Transient thermal guide 2019

[10] "Thermal Expansion/Contraction", accessed on Mar 17, 2021 https://www.flightmechanic.com/thermal-expansioncontraction/.

[11] "Thermal Conductivity of solids and Metals", accessed on Mar 17, 2021 https://www.nuclearpower.net/nuclear-engineering/heat-transfer/thermal-conduction/thermal-

conductivity/thermal-conductivity-of-solids-and-metals/. 